\title{
Formation Flight in Low-Earth-Orbit at 150 m Distance - AOCS In-Orbit Experience
}

\author{
Daniel Schulze ${ }^{1}$, Jaap Herman ${ }^{2}$ and Sebastian Löw ${ }^{3}$ \\ German Aerospace Center (DLR), Oberpfaffenhofen, 82234 Wessling, Germany
}

\begin{abstract}
AOCS operations for two satellites flying at $\sim 514 \mathrm{~km}$ altitude with unprecedented small separation of $150 \mathrm{~m}$ upwards pose various new challenges and require creative solutions. Several on-ground safety measures were installed, but due to the short reaction times emphasis always lies with the on-board handling of problems. This is achieved by implementation of a data link (one-way) between the satellites, by the design of a new AOCS safe mode that has no effect on the orbit, by the complete re-work of the TerraSAR-X FDIR concept (Fault Detection, Isolation and Recovery) and by autonomous formation control with a dedicated cold gas system on the second satellite TanDEM-X. This paper describes AOCS operations for the TanDEM-X mission in close formation. Most of the tasks are, thanks to extended safety measures and automated control mechanisms, routine. This will be described in the first section, where a short summary of all AOCS related operations will be given. The second section describes some special activities in connection with the cold gas system and the star trackers on TDX. The investigations and the fine tuning of the GPS receivers on both satellites are also described in this section. Although there was never a threat to the formation, close monitoring is required all the time and some mitigation measures were called for. Finally, the influence of the approaching solar maximum in 2013 will also be described in this section. The last part presents the conclusions and contains some suggestions for future missions.
\end{abstract}

\section{Nomenclature}

$\Delta \mathrm{v} \quad=$ increment in velocity

e $\quad=$ eccentricity

$\mathrm{i} \quad=$ inclination

\section{Introduction}

$\mathrm{T}_{\mathrm{C}}^{\mathrm{H}}$

HE TanDEM-X project is implemented by a "Public-Private Partnership" between the German Aerospace Centre (DLR) and Astrium GmbH.

The primary goal of the TanDEM-X (TerraSAR-X add-on for Digital Elevation Measurement) mission is to generate a global digital elevation model. To achieve this, two satellites - TerraSAR-X (TSX) and TanDEM-X (TDX), a satellite of almost identical construction - are forming the first configurable SAR (Synthetic Aperture Radar) interferometer in space with a separation of only a few hundred meters. A powerful ground segment, which is interlaced with that of TSX, completes the TanDEM-X system. The satellites are currently flying in close formation. Three years with various baselines will suffice to cover the entire surface of the Earth.

1 AOCS engineer, German Space Operations Center, Oberpfaffenhofen, 82234 Wessling, Germany/ D.Schulze@dlr.de

2 AOCS team lead, German Space Operations Center, Oberpfaffenhofen, 82234 Wessling, Germany/ Jaap.Herman@dlr.de

3 AOCS engineer, German Space Operations Center, Oberpfaffenhofen, 82234 Wessling, Germany/ Sebastian.Loew@dlr.de 
DLR is responsible for the scientific exploitation of the TSX/TDX data, as well as for planning and implementing the mission, for controlling the two satellites and for generating the digital elevation model. Astrium has built the satellites and shares in the costs of development and exploitation. As is the case already with TSX data, the responsibility for marketing the data lies in the hands of Infoterra $\mathrm{GmbH}$, a subsidiary of Astrium GmbH.

\section{A. TSX and TDX}

TSX was launched from Baikonur on June $15^{\text {th }}, 2007$ with a Dnepr rocket into a sun-synchronous dusk-dawn orbit at an altitude of $514 \mathrm{~km}$. The ground coverage is repeated in an eleven day cycle, which is achieved by controlling the spacecraft in a $\pm 250 \mathrm{~m}$ tube around a predefined Earthfixed reference orbit [6].

TDX was also launched from Baikonur almost exactly three years after TSX, namely on June $21^{\text {st }}, 2010$. The initial distance of $16000 \mathrm{~km}$ was soon reduced, a first formation at $<2 \mathrm{~km}$ was reached mid of October and the TanDEM-X mission started in December 2010 with an intersatellite distance of $\sim 300 \mathrm{~m}$ (see [7] for formation acquisition).

SAR data takes are usually made in "right-looking" mode under a $33.8^{\circ}$ angle towards the night side of the earth. Most of the time the satellite will have this attitude, but it is also possible to do a $67.6^{\circ}$ roll rotation in order to make a "left-looking"

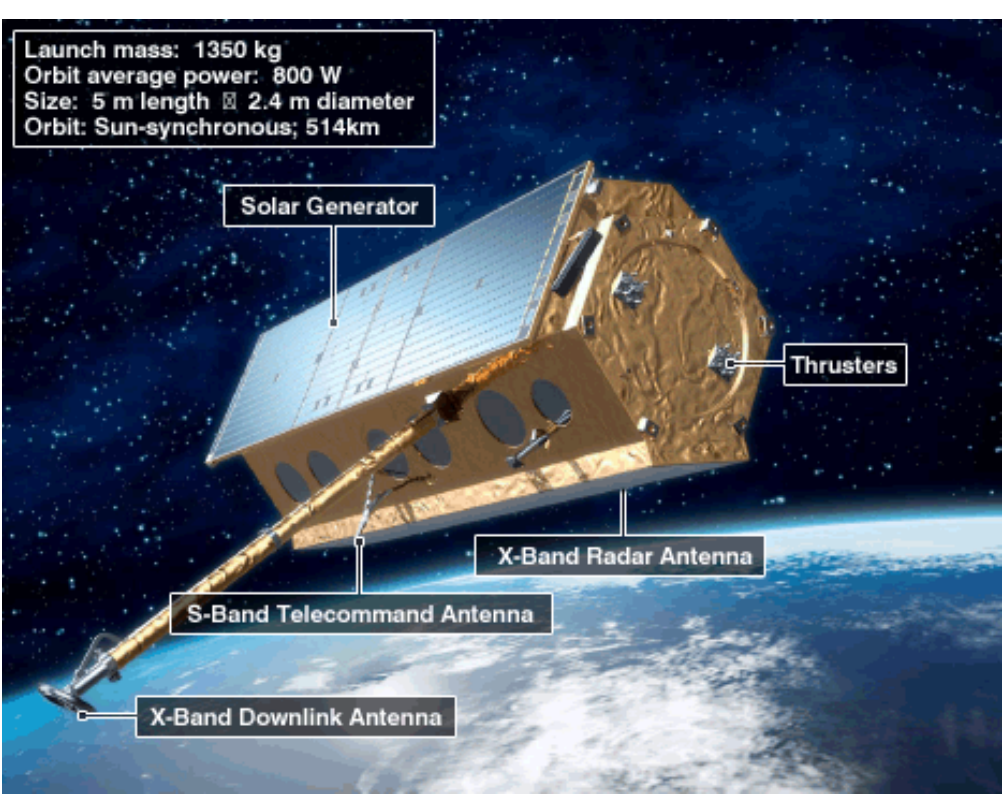

Figure 1. Illustration of TSX in space. The hydrazine thrusters are mounted on the back of the satellite. There is only one solar panel, which means that power problem can arise if the attitude is lost. data take. Images can be made in several instrument modes and spacecraft formations with ground resolution ranging from $\sim 0.5 \mathrm{~m}$ to $16 \mathrm{~m}$ and an area covered from $\sim 50 \mathrm{~km} 2$ to $104 \mathrm{~km} 2$. A more detailed description of the several options can be found in [5] and [9]. Both spacecraft are equipped with a hydrazine propulsion system with four redundant $1 \mathrm{~N}$ thrusters mounted at the rear side of the satellite (Fig. 1). Inclination maneuvers and maneuvers in anti-flight direction thus require a $\pm 90^{\circ}$ or $180^{\circ}$ yaw slew. Each thruster has a small tilt with respect to the spacecraft's length axis, so that by selecting various pairs the three satellite axes can be controlled independently. The spacecraft's attitude during routine operations is measured by star trackers (double redundancy) whereas attitude control is provided by four reaction wheels plus magnetic torque rods for wheel unloading (see [2] for a detailed description of all sensors and actuators).

The TDX satellite is in general a soft- and hardware rebuild of TSX, but some important changes were made in order to allow for close formation flight:

- a cold gas system with four redundant $0.04 \mathrm{~N}$ thrusters for formation keeping maneuvers, mounted at the front- and the rear-sides of the satellite

- $\quad$ an S-band receiver/decoder to handle telemetry from TSX; via an ISL (Inter Satellite Link) 120 parameters of TSX are made available on TDX

- algorithms for autonomous formation flight (TAFF); GPS measurements are used for orbit calculation and prediction as well as for the planning of cold gas maneuvers

\section{B. Formation Flight and Safety measures}

The request of a configurable close formation between TSX and TDX arises from the wish for a SAR interferometer in space. The satellites fly in almost identical orbits whereby the position of TDX describes a helix around the trajectory of TSX. This is achieved by separation of the relative eccentricity and inclination vector [4]. The maximal radial separation is reached over the poles (vertical baseline typically between $200 \mathrm{~m}$ and $500 \mathrm{~m}$ ) and the maximum separation in normal direction occurs at the equator (horizontal baseline typically $200-500 \mathrm{~m}$; see Fig. 2). This way it can be assured that the radial and normal separation never become zero at the same time. The 
shape of the helix depends upon the mission phase. The formation with the smallest baseline had a minimum separation of $150 \mathrm{~m}$. Orbit correction maneuvers are carried out with the hydrazine propulsion system simultaneously on both spacecraft with exactly the same $\Delta v$. Additionally formation keeping maneuvers are needed to compensate the drift of the relative e-vector that arises from the J2-perturbation [4]. These maneuvers are made only on TDX with the cold gas system.

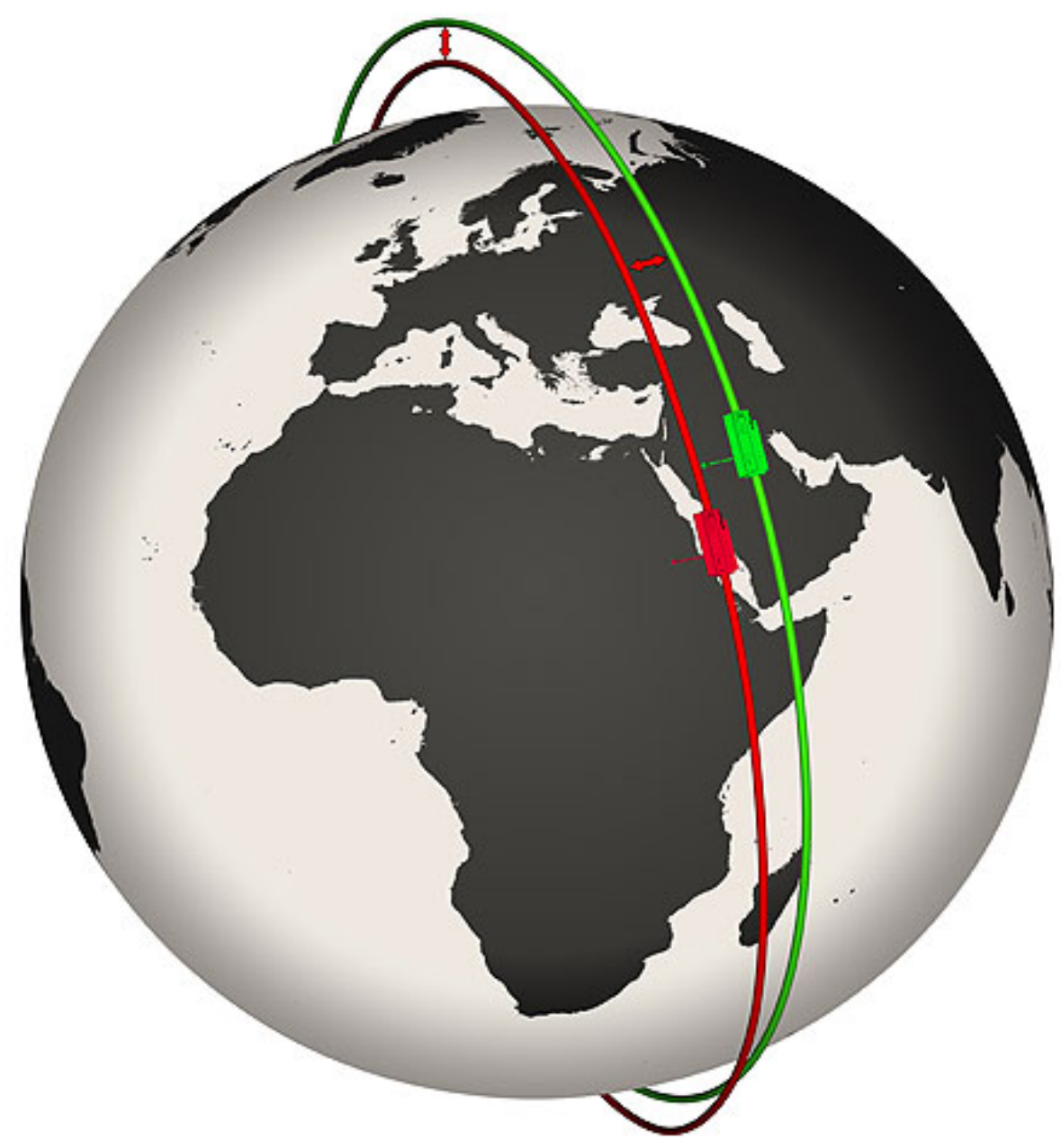

Figure 2. TSX and TDX flying in close formation. TDX (green) describes a helix around the orbit of TSX (red). The radial separation is largest over the poles and becomes zero at the equator, where the maximal separation in normal direction is found however. Typically, the distance between the satellites is between 150 and 500 meters.

Thrusters were originally planned to be the prime actuators during non-nominal situations in AOCS safe mode. Experience with TSX showed however, that the design with the thrusters mounted at the back of the satellite is far from ideal for flight in close formation. Analyses showed a collision risk of 1/500 due to orbit changes in case of a drop to the thruster based safe mode [6]. The reason is that just a minor part of the thrust is available for attitude control, whereas the major part is changing the orbit in an unpredictable way. Therefore, a second type of safe mode was implemented with the intention to control the attitude without changing the orbit. The so-called magnetorquer safe mode (ASM-MTQ) only uses the magnetic torque rods as actuators, whereas it still relies on CESS, magnetometer and IMU as sensors, just like the original thruster based safe mode (ASM-RCS). However, the 
damping of the rotation rates and the recovery of the attitude takes longer in ASM-MTQ than in ASM-RCS due to the weakness of the magnetic field at $514 \mathrm{~km}$ altitude. The maximum overall body rate that can be handled are $0.5 \% \mathrm{~s}$ due to the concept that the torque rods and the magnetometers are operated in alternation to allow disturbance free measurements of the Earth's magnetic field.

The new FDIR (Fault detection, Isolation and Recovery) design intends to always use the magnetorquer based safe mode first when a severe anomaly has been detected. There are performance limitations in ASM-MTQ as mentioned above, and it might still become necessary to make use of the conventional but more powerful ASMRCS. The latter will only be used if the continuation of the mission is seriously endangered. A possible scenario would be the battery voltage dropping below a certain value, a star tracker getting too hot or non-convergence of the attitude after three orbits. The thruster on-time is limited at first instance to make sure that the generated $\Delta v$ cannot lead to a collision of the satellites. A reboot of the on-board computer will follow in the worst case scenario when despite of limited use of the thrusters no convergence was reached. The spacecraft will come up after the reboot in ASM-MTQ again, but this time with wider power/thermal limits. The described sequence is tried only once however. If there is still no convergence or the power/thermal limits are yet violated, the spacecraft will be sent by FDIR to ASM-RCS once more, but this time without limitations to the thruster on-time. A more detailed description of the FDIR design implemented on TSX and TDX is found at [5].

The inter-satellite link is also used for surveillance, but is subject to some limitations. In the first place, the link only works in one direction and in the second, the connection is interrupted anytime the transmitter of TSX or TDX is switched to high-rate for ground station contacts. Therefore it is seen more as an extra safety than the part to completely rely upon. The ISL is used to transmit some essential parameters of TSX (including GPS position and velocity) to TDX in order to feed TAFF algorithms (Tandem Autonomous Formation Flight). A full description of the ISL can be found in [1] and a detailed description of the TAFF system is given in [3].

Close formation flight also poses the risk of mutual illumination to the spacecraft. In case one of the radar instruments is exposed to the signal of the one from the other spacecraft, a direct hit would be nine orders of magnitude higher than the usually measured field strength from back-scattering of the surface of the Earth. This could lead to a permanent damage of the radar instrument [10]. Therefore, it must be guaranteed that no data take is scheduled when one satellite is flying in the path of the radar beam of the sending spacecraft. This is done by introduction of so-called exclusion zones where data takes are not allowed and will be inhibited on-board if commanded from ground by mistake [10].

The described safety measures ensure that even without ground intervention the satellites remain in a safe and stable situation for at least ten hours. Nevertheless, ground station contacts for spacecraft monitoring are made every six hours to each spacecraft.

\section{Routine Operations}

\section{A. AOCS surveillance}

The most vital AOCS parameters, such as sensor performance, attitude errors, actuator commands, etc. are monitored on-board. In case of severe anomalies FDIR can react immediately and switch to the redundant hardware for instance. During ground station contacts a large number of parameters are checked in the mission control system against pre-defined limit settings and violations are indicated by yellow or red flags. The dump files (data covering also the time span in between ground station contacts) are screened with the same limit settings, and violations are reported by e-mail. The events will subsequently be analyzed and it is then decided if they can be disregarded or if a threat to the satellite is developing.

Several hundred configurable AOCS parameters that are constant for a longer time (like the moments of inertia for example) are checked once per week and compared to the currently desired setting. The same is done for the FDIR tables with the objective to notice commanding errors or bit-flips on short notice.

\section{B. Resources, maintenance and parameter adjustment}

Further routine tasks of the AOCS engineers are the calculation and administration of hydrazine and cold gas expenditure as well as the administration of thruster cycles. Based on the given budget the result is converted into an estimated mission lifetime. The amount of available cold gas at launch was just enough to meet the mission goals, because mass restrictions prevented a safety margin. Therefore, its monitoring is of prime interest. It has to last for at least three years of formation flight. Two different methods are used to derive the consumption. The latest plot for one of them (bookkeeping method) is shown in Fig. 3.

Long-term analyses and parameter adjustments are made for several AOCS parameters such as the CESS temperatures and reaction wheel friction. Also some FDIR limits require regular updates because of formation 
changes or due to pressure dependency. The allowed thruster on-time during the first entry in ASM-RCS is a good example, since it is depending on the current formation and the tank pressure. FDIR limits of the cold gas pressure on TDX also require updates to take the expenditure into account.

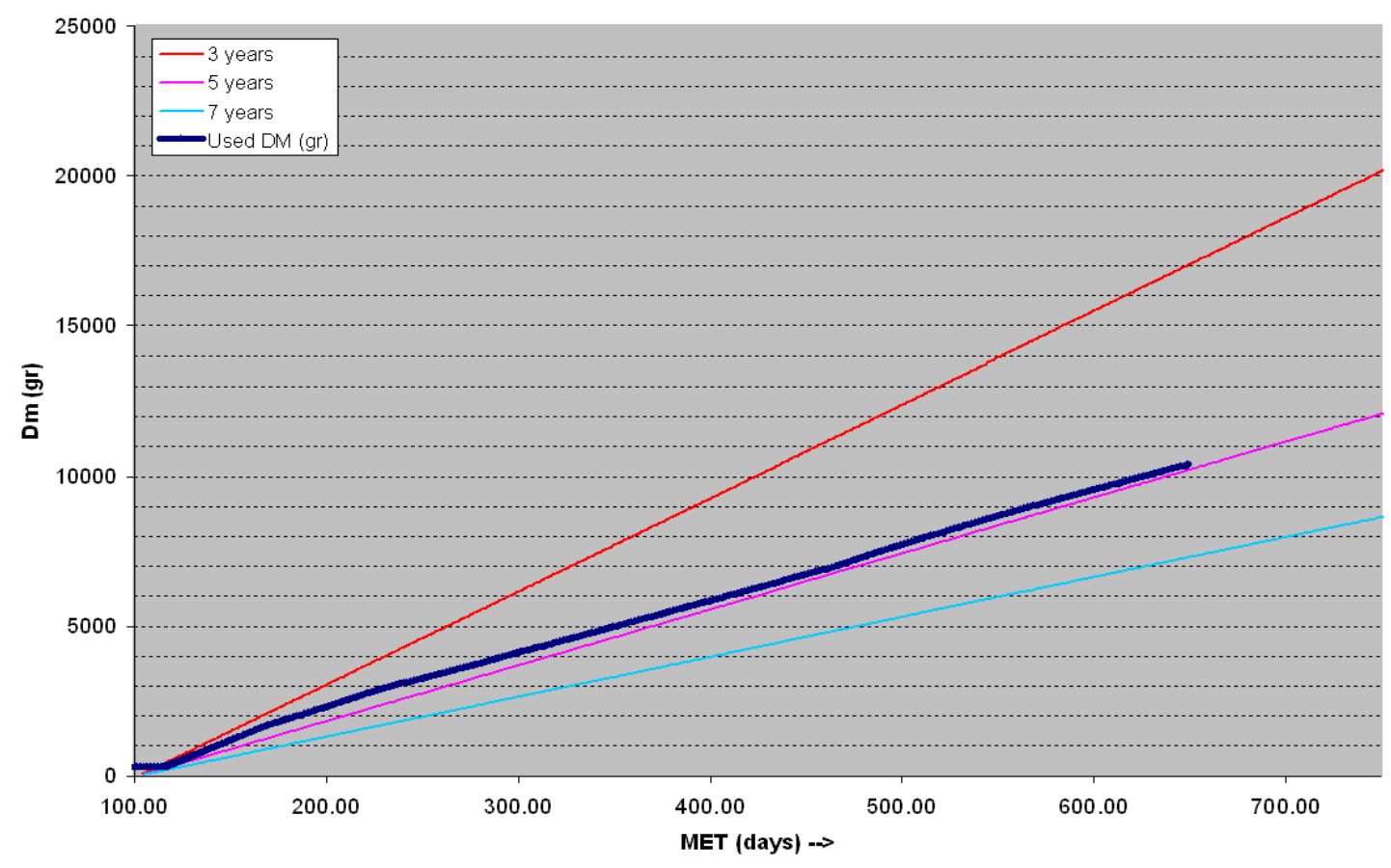

Figure 3. Used cold gas. The amount of actually expended cold gas (dark blue) is plotted over the mission elapsed time. The other lines show the expected consumption for different mission durations. It is seen that almost five years of formation flight could be achieved assuming a linear extrapolation.

\section{Maneuvers}

Hydrazine maneuvers are made on both satellites in order to maintain the reference orbit. The frequency and strength depend on the solar activity and ranges from one to four maneuvers every ten days with a strength between one and three $\mathrm{cm} / \mathrm{s}$. The variation is caused by solar activity, which influences the scale height of the atmosphere. The orbit corrections are calculated by the flight dynamics group and carried out simultaneously on both satellites. The command operators have to confirm the upload of both flight procedures via email. Nevertheless, the flight dynamics group also makes sure that a failed uplink or a failed execution on one satellite does not lead to a collision risk. There are also some provisions in place that protect against maneuver over-performance:

- The burn is limited to $110 \%$ of its desired burn duration (set within the AOCS flight procedure).

- A specific heater that is required to be on during the burn is switched off after a fixed time at the end of each maneuver. The related command is built in the AOCS flight procedure and would interrupt a still ongoing maneuver.

- The active propulsion branch will be switched off automatically in case a stuck open thruster is detected.

- Maneuvers on TDX will be inhibited in case the ISL indicates a problem in the propulsion system on TSX or if TSX is in safe mode

Cold gas maneuvers are made on TDX only. Once per 24 hours one maneuver pair is carried out, that consists of two maneuvers separated by half an orbit in order to correct the relative e-vector [8]. The 24 hour interval may be interrupted in case hydrazine maneuvers are made, which need to be calibrated first before new formation corrections can be planned. The maneuver size increases with the radial distance established in the current formation and typically $\Delta v$ values between 0.4 and $0.8 \mathrm{~cm} / \mathrm{s}$ in flight or anti-flight direction appear. Execution problems do not have such serious consequences as is the case for the hydrazine maneuvers. The formation is safe for at least 9 days without any maneuvers at all. Furthermore, the cold gas maneuvers are limited to burn durations of $300 \mathrm{~s}$ by FDIR. 


\section{TAFF - algorithms for autonomous formation flight}

The TAFF algorithms running on TDX provide a filtered navigation solution based upon TSX (via ISL) and TDX GPS data. The desired formation is stored in the on-board memory and can be changed by ground commands. If the deviation between the actual and the nominal formation is larger than a certain limit, warnings or alarms are indicated during ground station contacts.

TAFF was also designed to calculate formation keeping maneuvers based on navigation prediction and the desired formation. The maneuver planning is running constantly on-board TDX whereas the actual execution of these corrections is still prohibited and made by ground. TAFF is switched to "close loop" exclusively for test campaigns, where it is alone in control of the planning as well as the execution of formation keeping maneuvers. Results of the first close loop campaign are described at [3]. A second test campaign is scheduled for summer 2012.

\section{Special Operations and in-orbit Experience}

\section{A. Star trackers}

Both TSX and TDX have three star trackers mounted with different lines of sight. Two are operated in hot redundancy to protect against short outages and blindings from the Sun or the Moon. Sun blindings are not expected when the satellites fly in nominal attitude and the star camera boresights are perpendicular to the Sun. Moon blindings, however, can occur in principle, but have never been observed. Nevertheless, both spacecraft occasionally experience short outages from time to time with a period of one orbit (see Fig. 4). It was soon discovered that those events are caused by reflections of the other satellite in the field of view (FOV) of the affected star tracker. The duration of the blindings changes with time, because the relative geometry between the spacecraft and the sun varies slightly between the orbits.

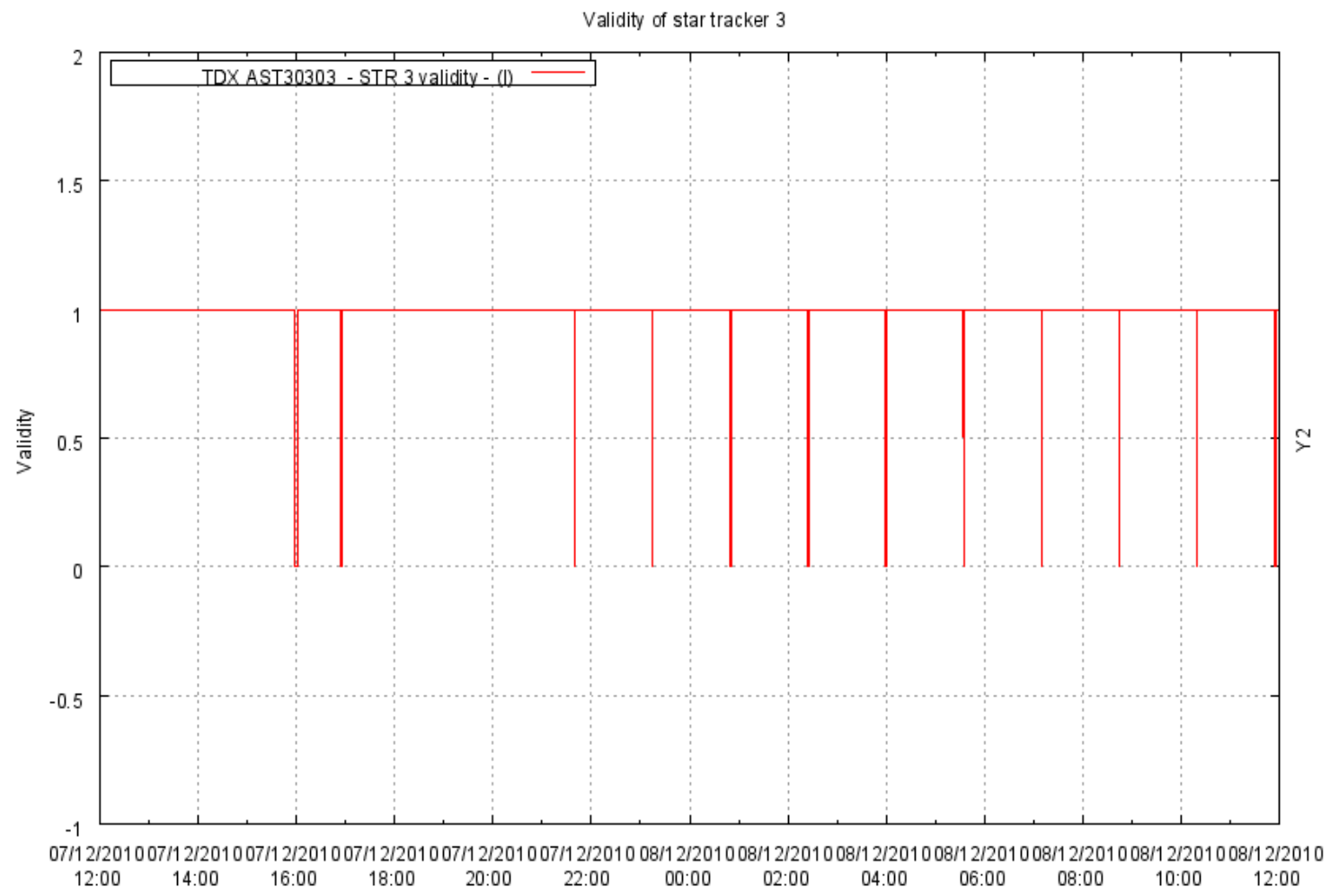

Figure 4. Plot of the validity flag (1=valid) of star tracker 3 on-board TDX. Outages are seen to occur with $a$ periodicity of one orbit (94 minutes). Analysis showed that TDX was flying over the South Pole during the events at a moment that TSX was in the field of view of star tracker 3. Blindings caused by TDX occurred also on TSX while flying over the North Pole. 
No actions had to be taken regarding the blindings. They took less than 20 seconds and were only observed on star tracker 3, which was used as cold backup as long as all three cameras were functional. Payload operations were not disturbed by the outages.

More outages, however, were observed on TDX than on TSX even at times where TSX was not in the FOV of the star tracker. All star cameras on-board TDX were affected by this behavior. A further analysis was requested from the manufacturer. The root cause was suggested to be the star camera's preprocessor, an additional functionality, which is available on TDX only. This assumption could be verified, because the outages disappeared during a test phase in which the preprocessor was disabled. All three preprocessors on TDX were disabled permanently by a s/w patch (a simple switch off is not sufficient because the preprocessing function would have been re-enabled after each power cycle).

After four months of formation flight a non-nominal behavior of star tracker 1 on TDX was observed. Investigations were made by GSOC and the manufacturer, but the root cause has not been fully identified yet. Thanks to the comfortable redundancy, it was possible to take the camera out of the AOCS control loop until analysis is finished. The star tracker is currently excluded in an on-board control procedure used by FDIR which switches on all cameras in case of missing attitude determination.

All taken measures were not strictly necessary for the success of the mission, but ensure the best possible performance of the star trackers on TDX.

\section{B. Cold gas system}

An unexpected reaction of the cold gas pressure in the active branch was noticed on September $17^{\text {th }}$, 2011. The cold gas system consists of a high pressure section and a low pressure section separated by a latch valve and a pressure regulator, which keeps the low pressure at a constant level of $\sim 2.8$ bars. During maneuvers, however, the pressure drops to 1.3 bar and recovers to 2.8 bar in the next 12 hours. After the second maneuver on the mentioned day the pressure only reached 2.1 bar and did not rise further. It was decided to carry out one more maneuver pair on the active branch hoping this would close a thruster. The situation did not change, however, and so the latch valve was closed and a switch from branch B to branch A was made. Fig. 5 shows a plot of the measurements of the low pressure transducers (LPT) of branch A (red) and B (green). After the latch valve was closed, the pressure in branch B dropped, but stabilized at $\sim 1.7$ bar for a while. This was caused by the high pressure gas that was hold in between the latch valve and the pressure regulator and was now released gradually into the outer section. About 12 hours later the regulator was completely open and no more gas was available to compensate the leaking. An exponential pressure drop is seen from there. The formation control maneuvers were resumed shortly after the branch switch and there is no impact on payload operations.

The flight dynamics group also observed an increase of two meters in the semi-major half-axis of TDX with respect to TSX over the time between the last two cold gas maneuver pairs with branch B. The amount of cold gas lost is estimated to be very small, namely 6 grams. Root cause is most probably a not perfectly closed thruster on the rear side of TDX originating from contamination of the thruster sealing surface with a small particle. The thruster manufacturer also suggested possible recovery actions that pursue the goal to flush any particle out of the sealing surface. It was decided to not execute this recommendation as long as the satellites fly in close formation and branch A is fully operational. Moreover, formation control is still possible by using branch B, but this would have two operational consequences. The small thrust generated by the leaking thruster must be considered in the maneuver planning and the cold gas expenditure would increase by about three grams per day (increase of $\sim 15 \%$ ) assuming the leakage will remain constant.

A further operational change made within the AOCS was the FDIR reconfiguration. An originally implemented autonomous switch from branch A to branch $\mathrm{B}$ is no longer foreseen in case of problems with branch A. 


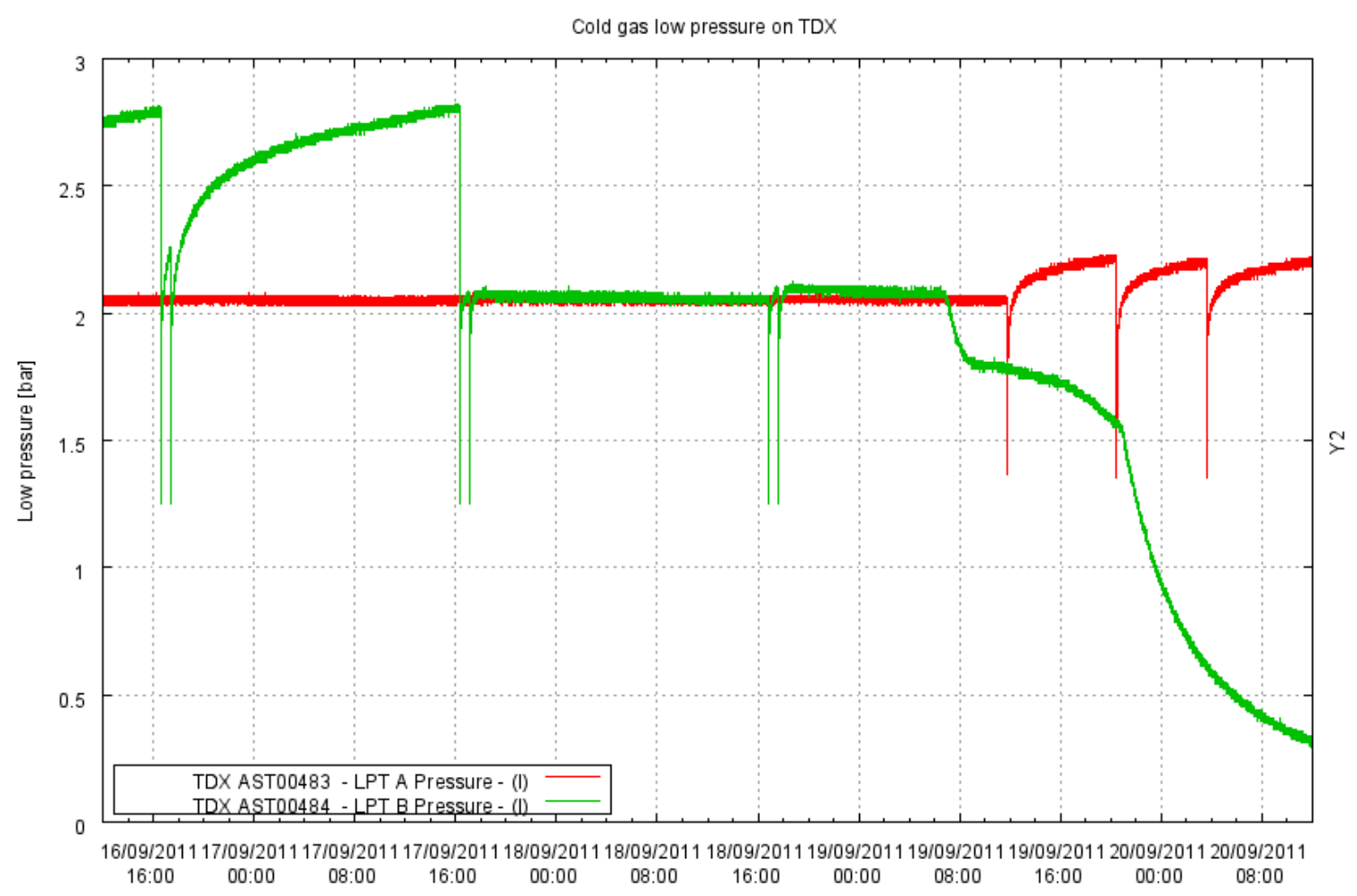

Figure 5. LPT (low-pressure transducer) values. The behavior of the low-pressure in branch $B$ which is shown above was detected after the maneuvers on Sep. 17 $7^{\text {th }}, 2011$ (second pair of vertical lines from the left). A switch to the other branch was made on Sep 19 $9^{\text {th }}$ at 06:50. The pressure on LPT B dropped quickly to 1.8 bar followed by a 12 hours compensation phase, where gas remaining between latch valve and pressure regulator was slowly released by the latter into the low pressure section. Finally, an exponential decrease is seen.

\section{GPS receiver}

There are two Mosaic receivers on-board TSX and TDX that are operated in cold redundancy. Occasionally performance degradation is observed, which can be analyzed because a second type of GPS receiver ("IGOR" Integrated GPS Occultation Receiver) is available aboard the spacecraft and is used as secondary payload for GPS occultation measurements ${ }^{4}$. This allows a direct comparison of the position solutions of both receivers to each other. The difference can be monitored in real time during contacts and also in the dump data. Several events with differences between Mosaic and IGOR receiver of up to a few hundred meters and durations between 5 and 30 minutes were observed and later analyzed in detail by the flight dynamics group. Their investigations showed which receiver is the one with problems. A deeper analysis by the manufacturer exposed a problem of the Mosaic measurements with signals from GPS vehicles with low elevation, more precisely with the processing of signals influenced by the atmosphere. Imprecise position and velocity information of the Mosaic receiver directly influences the solutions of the TAFF algorithms. The impact during the first TAFF close loop campaign is described in [3]. It was possible to inhibit the use of low elevation GPS space vehicles (elevation $<-7^{\circ}$ ) by reconfiguration of the Mosaic receiver settings. This clearly improved its performance. Orbit and formation control on ground is based upon data from the IGOR receiver and therefore not affected by inaccurate measurements of the Mosaic receiver. High accuracy GPS data are required only for autonomous formation flight and therefore routine payload operations are not affected.

Unforced reboots of the Mosaic receivers are another observation. Their influence, however, is minimized by the use of an on-board orbit propagation to bridge the gaps of normally less than two minutes.

${ }^{4}$ In fact, the IGOR receiver is also used for the precise orbit determination required for calibration of SAR data takes. 


\section{Influence of the solar activity}

Solar activity has an eleven year cycle. Some effects with impact on TSX and TDX are expected also with the approaching maximum in 2013. Ultraviolet flux from the Sun and disturbances in the Earth's magnetic field heat the upper atmosphere causing more air drag to satellites in low earth orbit. The increased scale height of the atmosphere results in an increase of the frequency as well as of the strength of orbit maintenance manoeuvres. In 2007 orbit corrections on TSX were required about once per 10 days, whereas in 2012 one maintenance maneuver was necessary every four days on average. An estimation taking the increasing propellant use into account shows that the hydrazine will suffice until at least 2016 on TSX and 2020 on TDX.

With respect to radiation effects on the spacecraft's hardware it was decided not to take any preventive measures such as switching off units in case of solar storms, but to rely on the on-board safety mechanisms. This could lead to an interruption of payload operations and might make recovery actions necessary. Preventive actions therefore focused on review and updates of AOCS flight procedures for contingency recovery.

\section{Conclusion}

It has been shown in the past one and a half years that routine operations in a close formation with inter-satellite distances of $150 \mathrm{~m}$ can be carried out successfully. Mandatory are close surveillance, good ground station coverage and a well designed FDIR concept on-board. An AOCS safe mode that does not influence the orbit and hence does not change the formation is a necessity. The especially designed "magnetorquer" safe mode was extensively tested in-orbit before the start of close formation.

Attitude and orbit control continue on both satellites in close formation without any problems or anomalies. All special AOCS activities were only necessary to optimize overall performance.

Safety and stability of such close formations could in future be enhanced by completely autonomous flight. Major restriction is the dependence upon the GPS receivers, which occasionally delivered imprecise results with deviations of a few hundred meters. It was shown that improvements of the receiver settings and a more robust configuration of TAFF algorithms could overcome intervals with such excursions and enable truly autonomous formation flight.

Tracking of available resources shows that the goal of three years of formation flight can be easily achieved and everybody in the project is feeling optimistic to complete the digital elevation model of the Earth on schedule.

The performance of the attitude and orbit control system has been shown to cope with the challenges of close formation flight in an excellent manner. This is mainly due to the good performance and design of the spacecraft's hard- and soft-ware. 


\section{Appendix A \\ Acronym List}

$\begin{array}{ll}\text { AOCS } & \text { Attitude and Orbit Control System } \\ \text { ASM-MTQ } & \text { Magnetorquer safe mode } \\ \text { ASM-RCS } & \text { Thruster safe mode } \\ \text { CESS } & \text { Coarse Earth and Sun Sensor } \\ \text { FOV } & \text { Field of View } \\ \text { IGOR } & \text { Integrated GPS Occultation Receiver } \\ \text { IMU } & \text { Inertial Measurement Unit } \\ \text { ISL } & \text { Inter Satellite Link } \\ \text { MET } & \text { Mission Elapsed Time } \\ \text { SAR } & \text { Synthetic Aperture Radar } \\ \text { TAFF } & \text { Tandem Autonomous Formation Flight } \\ \text { TDX } & \text { TanDEM-X Satellite } \\ \text { TSX } & \text { TerraSAR-X Satellite }\end{array}$

\section{Acknowledgments}

The authors wish to thank Andreas Spörl, Christoph Giese and Dieter Ulrich for their helpful comments and the critical reading of this article.

\section{References}

[1] TanDEM-X; Inter-Satellite Link - Decoder; TDX-AED-DD-0002; Astrium, 2007

[2] TanDEM-X: Design \& Interface Document; TDX-AED-DD-0001; Astrium, 2008

[3] Ardaens, J.S., D’Amico, S., Fischer, D.: “Early Flight Results from the TanDEM-X Autonomous Formation Flying System”; $4^{\text {th }}$ Spacecraft Formation Flying Missions \& Technologies Conference, St.Hubert, Canada, 2011

[4] D’Amico, S., Montenbruck, O.: "Proximity Operations of Formation-Flying Spacecraft using eccentricity/inclination vector separation”, Journal of Guidance, Control, and Dynamics, 2006

[5] Herman, J., Fischer, D., Schulze, D., Loew, S., Licht, M.; “AOCS for TanDEM-X - Formation Flight at $200 \mathrm{~m}$ Separation in low-Earth Orbit”; SpaceOps conference, Huntsville, USA, 2010

[6] Kahle, R.; “TSX/TDX Formation Collision \& Illumination Aspects”; TD-MOS-TN-4060; GSOC, 2008

[7] Kahle, R., Schlepp, B., Meissner, F., Kirschner, M., Kiehling, R.; "TerraSAR-X/ TanDEM-X Formation Acquisition Analysis and Flight Results”; $21^{\text {st }}$ AAS/AIAA Space Flight Mechanics meeting; New Orleans, USA, 2011

[8] Kahle, R.; “The TerraSAR-X / TanDEM-X Formation Flight: Challenges to Flight Dynamics and First Results"; $4^{\text {th }}$ Spacecraft Formation Flying Missions \& Technologies Conference, St.Hubert, Canada, 2011

[9] Krieger, G., Moreira, A., Fiedler, H., Hajnsek, I., Werner, M., Younis, M., Zink, M.; “TanDEM-X: A Satellite Formation for high-resolution SAR Interferometry”, IEEE Transaction on Geoscience and Remote Sensing, Vol. 45, No. 11, pp. 3317 - 3341, 2010

[10] Maurer, E., Zimmermann, S., Mrowka, F., Hofmann, H.: "Dual Satellite Operations in Close Formation Flight"; Proceedings SpaceOps 2012 , Stockholm, Sweden, 2012 